\title{
The Human Health Effects of Exposure to Polybrominated Biphenyls ${ }^{1}$
}

\author{
Jeoffrey K. Stross, ${ }^{*}$ Irving A. Smokler, $\dagger$ \\ JOHN ISBISTER, $\ddagger$ AND KENNETH R. WILCOX $\ddagger$ \\ *Departments of Internal Medicine and $\uparrow$ Psychiatry, University of Michigan Medical School \\ and $\ddagger$ The Michigan Department of Public Health, Ann Arbor, Michigan
}

Received September 11, 1980; accepted November 10, 1980

\begin{abstract}
The Human Health Effects of Exposure to Polybrominated Biphenyls. Stross, J. K., Smokler, I. A., Isbister, J., ANd Wilcox, K. R. (1981). Toxicol. Appl. Pharmacol. 58, 145-150. Polybrominated biphenyls (PBB) were inadvertently introduced into the food chain in Michigan in 1973. Fifty-one people with known exposure to PBB were studied. Twenty-three farmers with disabling health complaints, and 28 chemical workers involved in the PBB manufacturing process were systematically evaluated to determine if these highrisk groups suffered adverse effects from their known exposure to PBB. The farmers had a high frequency of constitutional symptoms, hepatomegaly and skin rashes, findings not commonly noted in the chemical workers. Biochemical and hematologic testing revealed few abnormalities, and electromyograms, nerve conduction velocities, endocrine studies, and lymphocyte transformation studies provided no objective findings that correlated with subjective complaints. There was no relationship between PBB levels and physical or laboratory abnormalities. Present evidence suggests that people exposed to PBB have few objective findings at this time, and reactive depression may be responsible for the high prevalence of constitutional symptoms.
\end{abstract}

In 1973 , between 500 and 2000 pounds of polybrominated biphenyls (PBB) were introduced into animal feed as a result of a shipping accident in Michigan (Carter, 1976). Farm animals who ingested this feed suffered various consequences, including weight loss, decreased productivity, skin changes, and reproductive abnormalities. Thousands of animals died or were destroyed, but widespread human exposure was most intense on quarantined farms (Humphrey and Hayner, 1975), and in workers involved in the PBB manufacturing process (Anderson et al., 1978); lowlevel exposure occurred throughout the state. A survey of breast milk samples obtained throughout the lower peninsula of

\footnotetext{
1 Presented in part at the American Federation for Clinical Research, May 7, 1979, Washington, D.C.
}

Michigan showed that 51 (96\%) of 53 samples contained detectable levels of PBB (Brilliant et al., 1976). A general population survey recently completed has revealed that only $10 \%$ of the population in Michigan does not have detectable levels of PBB in their blood (Selikoff and Anderson, 1979).

Polybrominated biphenyls are halogenated, aromatic hydrocarbons that were widely used as flame retardants in the plastic industries (Sanders, 1978). PBBs have low acute toxicity (Aftosmis et al., 1972), but are biologically persistent (Matthews et al., 1977), and are capable of causing chronic injury to the liver (Kimbrough et al., 1977), skin (Moorhead et al., 1978), kidneys (Moorhead et al., 1978), and lymphatic tissue (Luster et al., 1978). To determine the effects of PBB exposure on human health, the Michigan Department of Public 
TABLE 1

Historical Findings in Patients Exposed to PBB

\begin{tabular}{lcc}
\hline & $\begin{array}{c}\text { Farmers } \\
N=23(\%)\end{array}$ & $\begin{array}{c}\text { Chemical } \\
\text { workers } \\
N=28(\%)\end{array}$ \\
\hline Weakness & $23(100)$ & $3(11)$ \\
Fatigue & $22(96)$ & $3(11)$ \\
Arthralgias & $22(96)$ & $15(54)$ \\
Myalgias & $19(83)$ & $10(36)$ \\
Abdominal pain & $11(48)$ & $2(7)$ \\
Irregular bowel habits & $8(35)$ & $2(7)$ \\
Frequent infections & $11(48)$ & $3(11)$ \\
Skin rashes & $11(48)$ & $1(4)$ \\
Memory loss & $18(78)$ & $3(11)$ \\
Poor concentration & $22(96)$ & $3(11)$ \\
Irritability & $22(96)$ & $2(7)$ \\
Poor coordination & $7(39)$ & $1(4)$ \\
Hypersomnulence & $14(61)$ & $1(4)$ \\
Depression & $17(74)$ & $4(14)$ \\
\end{tabular}

${ }^{a}$ A high prevalence of historical complaints is noted in the farmer group and not found in the chemical workers.

Health, in conjunction with several federal agencies, established a cohort of 3600 persons with varying levels of PBB exposure. They were then surveyed to determine whether any changes in health status had occurred in relation to their PBB exposure. Although this cohort will be followed prospectively over the next several years, no associations were found between serum PBB levels and symptom prevalence rates and no statistically significant differences in lymphocyte number or function were noted (Landrigan et al., 1979).

While performing these studies, a subset of patients was identified who complained of multiple, disabling health problems. These people complained of a serious deterioration in their health status that corresponded temporarily to their known exposure to $\mathrm{PBB}$ and rendered them unable to engage in their previous occupations. Since no significant abnormalities were initially identified in the cohort of 3600 patients, as well as the subset of patients with the most profound health complaints, a more de- tailed study of this subset was undertaken to determine if their complaints could be related to PBB. A second group of patients was also studied, chemical workers who were involved in the manufacturing or distribution of PBB. These people had previously been found to have high levels of PBB in their serum and fat, and this study was aimed at determining whether high levels of PBB had led to adverse health effects. Since large-scale population studies have already been undertaken in the State of Michigan and have failed to show deleterious effects from $\mathrm{PBB}$; detailed, systematic evaluation of those with high levels of PBB and those with multiple complaints that were temporally related to their exposure were undertaken to define the potential health effects of PBB.

\section{METHODS}

Twenty-three persons, predominantly farmers from quarantined farms, with known exposure to $\mathrm{PBB}$ and incapacitating health complaints, were identified in the original study and asked to participate in a project aimed at determining the health effects of PBB. Twenty-eight workers from the Michigan Chemical Company, all of whom were involved in the manufacturing or distribution of PBB, were also evaluated at University Hospital. These workers were selected at random from a pool of 100 people known to have worked directly with PBB during the previous five years.

These 51 patients were evaluated in the Clinical Research Center of University Hospital. The evaluation of each patient included a history, physical examination, X-ray film of the chest, an electrocardiogram, and extensive biochemical and hematologic studies. Fasting blood specimens for baseline determinations of thyroid, adrenal, and pituitary function were obtained as were responses to induced hypoglycemia and thyroid-releasing hormone. Immunologic studies included quantitative determinations of immunoglobulin levels, skin testing, and lymphocyte transformation studies. Peripheral blood lymphocytes were studied for transformation to three mitogens: phytohemagglutinin (PHA), concanavallin A (Con A), and pokeweed mitogen (PWM), in wells of flat-bottomed microtiter plates. Each mitogen was run in quadruplicate wells that contained $10^{5}$ lymphocytes per well in a total volume of $0.2 \mathrm{ml}$ RMP1-1640 with $20 \%$ autologous 
plasma. Microtiter plates were placed in a $5 \% \mathrm{CO}_{2}$ humidified incubator at $37^{\circ} \mathrm{C}$. After incubation for $96 \mathrm{hr}$, cells were pulsed with $1 \mu \mathrm{Ci}$ of $\left[{ }^{3} \mathrm{H}\right]$ thymidine and harvested $6 \mathrm{hr}$ later with an Otto-Hillier cell harvester. Cells were then washed with saline, dried, resuspended in scintillation fluid, and counted. Data were expressed as mean counts per minute (cpm) per $10^{3}$ lymphocytes.

PBB analyses were performed on serum, feces, fat specimens obtained by open biopsy, and bile obtained by duodenal intubation. Creatine phosphokinase, aldolase, rheumatoid factor, antinuclear antibodies, and LE preparations were routinely obtained. Electromyograms and nerve conduction velocities, using a technique modified from Hopf, were also performed on each patient.

Patients underwent either psychiatric interviews or psychological testing. The psychological measures included Wechsler Adult Intelligence Scale, Minnesota Multiphasis Personality Inventory (MMPI), Beck Depression Inventory, Revised Visual Retention Test, Trail Making Test, Purdue Pegboard, Finger Tapping Test, Finger-Tip Writing Test, Babcock Story Recall Test, Memory for Unrelated Sentences Test, PairedAssociated Memory Test, and Rorschach.

\section{RESULTS}

In the farmer group, there were 19 males (83\%) and 4 females (17\%). Their ages ranged from 16 to 59 with a mean of 38 . The chemical workers consisted of 24 males $(86 \%)$ and 4 females (14\%) with ages of 23 to 62 and a mean of 40 years.

A characteristic symptom complex was apparent in the farmers but was not noted in the chemical workers. These symptoms are noted in Table 1 and are divided into five groups: general, musculoskeletal, cutaneous, abdominal, and neuropsychiatric.

The physical examination demonstrated two findings of interest in the farmers. The first was the relatively high prevalence of hepatomegaly. Eight patients (35\%) had evidence of liver enlargement defined as greater than $11 \mathrm{~cm}$ of vertical height in the midclavicular line. Liver scanning confirmed the presence of hepatomegaly in four of these individuals (17\%), and two of them had a history of substantial alcohol intake. Ten individuals (43\%) had skin le- sions, but these were common dermatologic problems, such as superficial mycoses and actinic keratosis, which are not uncommon in the general population and in people working outdoors. There were no other consistent findings noted in the farmers. The only abnormality noted in the chemical workers was the presence of hepatomegaly in two patients (7\%), both of whom heavily indulged in alcoholic beverages, and abnormal skin examination in four patients (14\%).

Numerous biochemical tests were performed, but the only abnormalities found were minor elevations of the serum uric acid, serum iron, serum cholesterol, and triglycerides. The serum uric acid, serum iron, and serum cholesterol elevations were found in $20 \%$ of the farmers and chemical workers and were only slightly above the normal range. Elevation of the triglyceride level was noted in $50 \%$ of the farmers and chemical workers, with a mean of $185 \mathrm{mg} \%$ (SD $\pm 50 \mathrm{mg}$ ), with an upper limit of normal of $150 \mathrm{mg} \%$. Abnormalities in liver function tests were noted in $4 \%$ of the patients, and there was no correlation between liver size and enzyme elevations except in those patients with a large intake of alcoholic beverages. PBB levels in serum and bile were similar and averaged 0.014 parts per million in the farmers and 0.048 parts per million in the chemical workers. Fecal levels were low and of no benefit. Fat levels averaged 3.26 parts per million in the farmers and 12.82 parts per million in the chemical workers. The partition coefficient between serum and fat of 250 is similar to what has been found in other studies. When patients were divided according to fat levels into high $(>50 \mathrm{ppm})$, moderate $(10-$ $50)$, and low PBB exposure $(<10)$, there was equal distribution of abnormal physical, laboratory, and diagnostic studies. This is in agreement with the earlier finding of no association between PBB levels and symptom prevalence rates (Landrigan et al., 1979). 
TABLE 2

Lymphocyte Function in Patients Exposed to PBB ${ }^{a}$

\begin{tabular}{|c|c|c|c|}
\hline & $\begin{array}{l}\text { Farmers } \\
N=23\end{array}$ & $\begin{array}{c}\text { Chemical workers } \\
\qquad N=28\end{array}$ & $\begin{array}{c}\text { MDPH staff } \\
N=9\end{array}$ \\
\hline Mean serum PBB level (ppm) & 0.014 & 0.048 & 0.002 \\
\hline Percentage of $T$ cells & 63.2 & 65.3 & 72.9 \\
\hline Percentage of B cells & 5.8 & 5.6 & 6.4 \\
\hline \multicolumn{4}{|l|}{$\begin{array}{l}\text { Maximal blastogenic responses } \\
\text { of blood lymphocytes } \\
\text { (scintillation cpm) }\end{array}$} \\
\hline PHA (Mean \pm 1 SD) & $42,278 \pm 20,202(p<0.05)^{b}$ & $58,136 \pm 25,562$ (N.S.) ${ }^{r}$ & $63,170 \pm 16,308(p<0.01)^{d}$ \\
\hline PWM (Mean \pm 1 SD) & $48,054=16,917(p<0.01)^{0}$ & $50,369 \pm 18,667(p<0.01)^{c}$ & $86,763 \pm 25,814(p<0.01)^{\prime}$ \\
\hline Con $A($ Mean $\pm 1 S D)$ & $54,187 \pm 24,861(\text { N.S. })^{b}$ & $47,888 \pm 17,652$ (N.S.) ${ }^{c}$ & $65,858 \pm 37,265$ (N.S.) ${ }^{d}$ \\
\hline
\end{tabular}

${ }^{a}$ Although statistically significant differences are noted between each group, all figures are in the normal range for this laboratory and these differences have no known biologic significance.

${ }^{b}$ 'Student's $t$ test-between farmers and chemical workers.

c Student's $t$ test-between chemical workers and MDPH.

a Student's $t$ test-between MDPH and farmers.

Most other diagnostic studies were normal aside from an occasional patient with an abnormal test result. Electromyograms and nerve conduction velocities revealed a slight decrease in nerve conduction velocities in three patients who were asymptomatic. Pituitary function studies were normal in all patients.

Because of reported abnormalities in lymphocyte numbers and function, these patients provided us with an additional opportunity to study patients under ideal circumstances. Blood specimens were drawn and transported to the laboratory where the studies were immediately initiated. Blood specimens obtained from Michigan Department of Public Health employees were used as controls. No abnormalities in lymphocyte number or function could be determined in these groups of patients (Table 2). Although statistically significant differences were noted in the blastogenic responses, all values are within the normal range for this laboratory and these differences have no known biologic significance.

Psychiatric evaluation revealed a high prevalence of depression. Eighteen (78\%) of the farmers had findings characteristic of reactive depression, but multiple tests of intelligence, memory, and functional ability failed to demonstrate abnormalities. Only six patients $(21 \%)$ in the chemical worker group had similar findings $\left(x^{2}=p<0.001\right)$. The depression related to their health complaints, financial difficulties, and their perceived problems in obtaining help and in more patients was improving over time.

\section{DISCUSSION}

Since the contamination of animal feed with PBB was discovered, a variety of studies have been undertaken to determine if PBB has caused any adverse health effects. The first study was carried out by the Michigan Department of Public Health, and it failed to identify any relationship between PBB levels and the presence or absence of symptoms. Abnormalities in lymphocyte function have been found by some workers (Bekesi et al., 1978) but could not be confirmed by others (Landrigan et al., 1979). A similar situation exists with the neurologic examination (Valciukas et al., 1979; Stross et al., 1979). A large population study is complete now and no serious abnormalities have been reported (Selikoff and Anderson, 1979).

This study concentrated on two highly selected groups of patients, one with multi- 
ple health-care complaints that began after the PBB contamination, and the other with high levels of PBB in their bodies. It was based on the premise that if PBB was going to cause toxic effects, they should be dose related, and those with high levels should be at greater risk. Since previous studies failed to reveal any dose relationship, we initiated a more comprehensive evaluation to determine if any abnormalities existed. We also studied those patients with multiple symptoms to determine if they could be related to their known exposure to PBB.

It is not surprising that the farmers had a high prevalence of historical complaints since they were selected because of them. The chemical workers had very few complaints, but since this study was undertaken at a time when a decision to close the plant was being considered, employees could have minimized their complaints. In spite of the frequent complaints of the farmers, there were few findings on physical examination and few abnormalities in their laboratory tests that corresponded to their complaints. Although one-third had an enlarged liver on examination, half of these were not confirmed by liver scan, thus, showing the unreliability of the physical examination for demonstrating hepatomegaly. There were two patients with proven hepatomegaly that could not be explained on the basis of other causes. Since both of these individuals were also in contact with multiple other chemical agents that are known hepatotoxins, it is not possible to state that a cause and effect relationship exists. Although skin problems were common, there were no cases of chloracne. No significant historical or physical findings were noted in the chemical workers.

Despite extensive biochemical testing, few abnormalities were found. Elevation in the serum triglycerides was the most common finding, averaging $185 \mathrm{mg} \%$, slightly over the normal value of $150 \mathrm{mg} \%$. Since Bekesi et al. (1978) reported abnormal lymphocyte function and numbers, and
Landrigan et al. (1979) were unable to confirm these findings, this study provides additional evidence that abnormal lymphocyte function is not commonly found. No anergy to skin testing was noted and quantitative determinations of immunoglobulin levels were normal. Many patients had musculoskeletal complaints but serologic studies failed to reveal evidence of rheumatic diseases, except in one individual with active rheumatoid arthritis. Joint $\mathrm{X}$-rays were normal or showed evidence of degenerative changes. Since peripheral neuropathies resulting from toxic exposures may preferentially affect slow-conducting nerve fibers, we measured the full range of conduction and failed to demonstrate any abnormalities.

The PBB levels of this group of chemical workers were identical to those previously found in Michigan Chemical Company employees, while the levels of the farmers were far lower but still in a range that was higher than the population at large in the state. There was an equal distribution of physical and laboratory abnormalities in people with low, moderate, or high PBB levels.

The most prominent findings were noted in the psychiatric evaluation and in the psychological testing. Objective evidence of organic change in the central nervous system could not be documented. Contrary to other reported studies (Brown and Nixon, 1979), tests of intelligence, memory, and fine motor control were normal despite a high prevalence of complaints relative to these areas. Since no changes could be identified in these high-risk groups, it is unlikely that they would be found in less exposed groups, such as the public at large. The pertinent findings were a high prevalence of depression and a somatic preoccupation about health. This was noted in almost $80 \%$ of the farmers versus $21 \%$ of the chemical workers. There were many factors that could have influenced this, including financial difficulties, legal proceedings, 
inability to obtain help in dealing with their problems, and a perceived coverup of the entire incident.

In conclusion, patients with multiple health-care complaints and known exposure to $\mathrm{PBB}$ and patients with high levels of PBB in their bodies were not found to have objective evidence of deleterious effects at this time. The most common finding to date is the presence of reactive depression which appears to have resulted from a myriad of problems surrounding the incident. Since animal studies have identified multiple serious problems, long-term follow-up will be needed to screen for those potential effects.

\section{ACKNOWLEDGMENT}

The authors thank the Michigan Department of Public Health for their assistance in patient selection, PBB analysis, and financial support for this project.

\section{REFERENCES}

Aftosmis, J. G., Culik. R., Lee, K. P., Sherman, H., AND WARITZ, R. S. (1972). Toxicology of brominated biphenyls. I. Oral toxicity and embroyotoxicity. Toxical Appl. Pharmacol. 22, 316.

Anderson, H. A., WolfF, M. S., Fischbein, A., AND Selikoff, I. J. (1978). Investigation of the health status of Michigan Chemical Corporation employees. Environ. Health Perspect. 23, 187-192.

Bekesi, J. G., Holland, J. F., Anderson, H. A., Fischbein, A. S., Rom, W., WolfF, M. S., AND SELIKOFF, I. J. (1978). Lymphocyte function of Michigan dairy farmers exposed to polybrominated biphenyls. Science 199, 1207-1209.

Brilliant, L. B., VANAmburg, G., Isbister, J., Humphrey, H., WilcoX, K., Eyster, J., Bloomer, A. W., ANd Price, J. (1976). Breast milk monitoring to measure Michigan's contamination with polybrominated biphenyls. Lancet 2, 673-676.

BRown, G. G., AND NIxON, R. (1979). Exposure to polybrominated biphenyls. J. Amer. Med. Assoc. 242, 523-527.

CARTER, L. J. (1976). Michigan's PBB incident: Chemical mix-up leads to disaster. Science 192, 240-243.

Humphrey, H. E. B., and Hayner, N. S. (1975). Polybrominated biphenyls: An agricultural incident and its consequences, an epidemiological investigation of human exposure. Presented at Ninth Annual Conference on Trace Substances in Environmental Health, Columbia.

Jackson, T. F., AND Halbert, F. L. (1977). A toxic syndrome associated with the feeding of polybrominated biphenyl contaminated protein concentrates to dairy cattle. J. Amer. Vet. Med. Assoc. $165,437-439$.

Kimbrough, R. D., Burse, V. W., and Lidde, J. A. (1977). Toxicity of polybrominated biphenyl. Lancet 2, 602-603.

Landrigan, P. J., Wilcox, K. R., Silva, J., Humphrey, H. E. B., Kauffman, C., and Heath, C. W. (1979). A cohort study of Michigan residents exposed to polybrominated biphenyls: Epidemiologic and immunologic findings. N.Y. Acad. Sci. 320, 284-294.

Luster, M., FAITH, R. E., AND MoORE, J. A. (1978). Effects of polybrominated biphenyls on immune response. Environ. Health Perspect. 23, 227-232.

Matthews, H. B., Kato, S., Morales, N. M., and TUEY, D. B. (1977). Distribution and excretion of 2, 4, 5, 2, 7, 5 hexabromobiphenyl, the major component of Firemaster BP-6. J. Toxical Environ. Health 3, 599-605.

Moorhead, P. D., Willett, L. B., and SchanBOCHER, F. L. (1978). Effects of PBB on cattle. II. Gross pathology and histopathology. Environ. Health Perspect. 23, 11-118.

SANDERS, H. J. (1978). Flame retardants-special report. Chem. Eng. News 22-36.

Selikoff, I. J., AND ANDERson, H. A. (1979). A survey of the general population of Michigan for health effects of PBB exposure. Submitted to the Michigan Dept. of Public Health.

Stross, J. K., Nixon, R. K., AND Anderson, M. D. (1979). Neuropsychiatric findings in patients exposed to polybrominated biphenyls. New York Acad. Sci. 320, 368-372.

Valciukas, J. A., Lilis, R., Anderson, H. A., WolfF, M. S., ANd Petrocci, M. (1979). The neurotoxicity of polybrominated biphenyls: Results of a medical field survey. New York Acad. Sci. 320, 337367. 